\title{
The Hot Hand Exists in Volleyball and Is Used for Allocation Decisions
}

\author{
Markus Raab \\ German Sport University Cologne and \\ Max Planck Institute for Human Development \\ Gerd Gigerenzer \\ Max Planck Institute for Human Development
}

Bartosz Gula

University of Klagenfurt

\begin{abstract}
The "hot hand" belief in sports refers to the conviction that a player has a higher chance of making a shot after two or three successful shots than after two or three misses (resulting in "streaks"). This belief is usually considered a cognitive fallacy, although it has been conjectured that in basketball the defense will attack a "hot" player and prevent streaks from occurring. To address this argument, we provide the first study on the hot hand in volleyball, where the net limits direct defensive counterstrategies, meaning that streaks can more likely emerge if a player is hot. We first establish that athletes believe in the hot hand in volleyball (Study 1A). Analyzing the top 26 first-division players, we then show that streaks do exist for half of the players (Study 1B). Coaches can detect players' performance variability and use it to make strategic decisions (Study 2A). Playmakers are also sensitive to streaks and rely on them when deciding to whom to allocate the ball (Study 2B). We conclude that for volleyball the hot hand exists, coaches and playmakers are able to detect it, and playmakers tend to use it "adaptively," which results in more hits for a team.
\end{abstract}

Keywords: decision making, streaks, sports

Many people who participate in, watch, or report on sports believe that a player has a higher chance of making a shot after two or three successful shots (hits) than after two or three unsuccessful shots (misses). One might hear a reporter say that "Lincy is a streaky shooter," for instance, or a fan claim that "the Lakers have a run." Such convictions based on the sequential performance of a player or team have been termed the hot hand belief (Gilovich, Vallone, \& Tversky, 1985).

In this article, we pose two questions. First, does the hot hand belief reflect reality? Second, how is the belief used for decision making? The first question seems to have already been answered in the literature, but a closer look shows that the answer is not clear-cut. One complicating factor is that the "hotness" of a player may not be observable because the other team increases its defense

This article was published Online First October 17, 2011.

Markus Raab, Institute of Psychology, German Sport University Cologne, Cologne, Germany and Center for Adaptive Behavior and Cognition, Max Planck Institute for Human Development, Berlin, Germany; Bartosz Gula, Department of Psychology, University of Klagenfurt, Klagenfurt, Austria; Gerd Gigerenzer, Center for Adaptive Behavior and Cognition, Max Planck Institute for Human Development.

We thank Anita Todd and Rona Unrau for their help in editing the manuscript, Uwe Czienskowski for programming the experimental paradigm, the Institute for Applied Training Sciences in Leipzig for providing video sequences of volleyball players, Ilka Gleibs and Jörn Köppen for their help in collecting and analyzing the questionnaires, and Joe Johnson, Jonathan Nelson, and Oliver Vitouch for valuable comments on a previous version of this article.

Correspondence concerning this article should be addressed to Markus Raab, German Sport University Cologne, Am Sportpark Müngersdorf 6, 50933 Cologne, Germany. E-mail: raab@dshs-koeln.de against the player, as, for instance, in basketball. Thus, finding no streaks can be the result of increased defense rather than merely the absence of the hot hand. In the following, we concentrate on a sport that limits this confounding factor: volleyball, where each team remains on a different side of the court. The second question is new, and in our view the more important one. We argue that players use the belief about streaks to alter their allocation behavior. In most team sports, one key strategy is to allocate the ball to the player with the strongest chance of scoring. It is argued that the belief in the hot hand can enhance the chance of scoring and thus may even be adaptive (Burns, 2004). If the hot hand belief is adaptive, then playmakers acting on it will increase the team's chance of winning, for instance, by allocating the ball more often to the player with the higher base rate or a current streak.

This is the first study on the hot hand in volleyball, and we show that (i) coaches, playmakers, and fans are sensitive to streaks, which reinforces the existence of the hot hand belief; that (ii) the hot hand exists for half of the players in our study; and (iii) this belief is used to guide playmakers' allocation decisions.

\section{State of the Art}

There is a huge body of literature on the hot hand phenomenon, virtually all of which has addressed the first question concerning its existence but not the second concerning its behavioral use. Belief in the hot hand is widespread, according to the first major experimental investigation by Gilovich and colleagues (1985) as well as subsequent studies in specific sport disciplines (baseball: Crothers, 1998; basketball: McCallum, 1993; Wolff, 1998; tennis: Klaasen \& Magnus, 2001; golf: Clark, 2003; Gilden \& Wilson, 1995b; horseshoe pitching: Smith, 2003; bowling: DorseyPalmateer \& Smith, 2004), sport science (Gould, Tammen, Mur- 
phy, \& May, 1991; Hales, 1999), perception (Gilden \& Wilson, 1995a), psychology (Adams, 1995; Larkey, Kadane, \& Smith, 2004; Oskarsson, Van Boven, McClelland, \& Hastie, 2009), and economics (Camerer, 1989). Although the unanimous conclusion is that the hot hand belief does exist, some, following Gilovich et al.'s original study, argue that "hotness" itself does not exist, whereas others maintain that it does (e.g., Larkey, Smith, \& Kadane, 1989). According to the review by Bar-Eli, Avugos, and Raab (2006), the score between these two camps in sports is nearly tied at $14: 13$.

Gilovich et al. (1985) initiated the debate about the hot hand belief with a set of studies showing that in basketball, individual sequences of hits and misses in field goals are nondependent (Gilovich et al., Study 2) yet that fans nevertheless believe in it (Gilovich et al., Study 1). Furthermore, they showed that independence of shots exists in free shots of professional basketball players (Gilovich et al., Study 3) and in a controlled free-shooting experiment of intercollegiate basketball teams (Gilovich et al., Study 4).

In one of Gilovich et al.'s surveys, 91 of 100 basketball fans believed that a hypothetical player has "a better chance of making a shot after having just made his last two or three shots than he does after having just missed his last two or three shots" (p. 297). The average response was that the chance of another hit was $61 \%$ if the player had made the last shots, compared with $42 \%$ if he had missed. This difference is the empirical observation that has been labeled the hot hand belief. Further research supported the definition of a streak based on at least three hits (e.g., Carlson \& Shu, 2007; Gula \& Köppen, 2009), which we will apply in our investigation. Belief in the hot hand was originally attributed to a "misperception of random sequences," assuming that some comparison between an observed sequence of hits and misses and a subjective notion of randomness is performed. Little is known about this process and how to measure streaks.

Comparing the conditional probability of hits after misses to that of hits after hits is one measure for the hot hand. Two others are runs and autocorrelations. A run is defined in the hot hand literature as three or more hits in a sequence (Carlson \& Shu, 2007). Imagine a player with a base rate of .5 (e.g., five of 10 shots are hits) who plays 16 rounds of four shots. We expect only one of 16 rounds to have four hits in a row $\left(1 / 2^{4}=1 / 16\right)$. If a player exhibits fewer runs than expected by chance, the conclusion is that this player has a hot hand (Hales, 1999). A third way to test the existence of a hot hand is autocorrelation (Bar-Eli et al., 2006). Imagine a player who makes hits $(\mathrm{H})$ and misses $(\mathrm{M})$ in the sequence MMHHHHHHMM with a base rate of .60 (six hits of 10). Autocorrelation counts the correlations between successive events; only in the third and ninth position in the given example does the following event ( $\mathrm{H}$ and $\mathrm{M}$, respectively) differ from the preceding event. The lag 1 autocorrelation is a correlation between the original sequence and the sequence moved by one position. If systematic autocorrelations exist, there is evidence of a hot hand.

\section{Cognitive Fallacy or Adaptive Behavior?}

Early researchers termed the hot hand belief a fallacy ${ }^{1}$ because successive shots were found to be independent (Gilovich et al., 1985) and thus players would be no more likely to hit after several hits than after several misses. From this, the conclusion was drawn that if people rely on wrong beliefs, it will be costly for, say, a team (Gilovich et al., 1985) or an individual placing bets (Camerer, 1989). This assumption is highly plausible, but is it correct?

Let us consider the alternative conclusion: Can false beliefs ever lead to advantageous behavior? For example, consider "as-if" theories in science, the practice of drawing the right conclusion from the wrong assumptions about underlying processes. A number of theories have postulated that cognitive limitations (Hertwig \& Todd, 2003; Kareev, 2000), "improper models" (Dawes, 1979), forgetting (Schooler \& Hertwig, 2005), and other apparent shortcomings can in fact improve behavior. The importance of judging cognitive processes by adaptive criteria, such as success and speed, is the focus of research on ecological rationality (Gigerenzer, Todd, \& the ABC Research Group, 1999). The logical question of whether a belief matches reality should not be confused with the ecological question of how useful the belief is to achieve some goal (Burns, 2004; Gula \& Raab, 2004; Hahn \& Warren, 2009).

To answer this ecological question, consider the case of two players, one with a base rate of .60 (player A) and the other with a base rate of .40 (player B). A playmaker could use many allocation strategies, such as probability matching (Gaissmaier \& Schooler, 2008; Gallistel, 1993). Probability matching would mean that of 100 balls, 60 are allocated to player A and 40 to player B, resulting in an expected value of $(60 \times .6)+(40 \times .4)=$ 52 successful hits out of 100 attempts. The hot hand belief is adaptive if players' performance changes in a systematic way such that momentary increases in performance relative to a player's average performance allow more balls to be allocated to him than to another player and thereby increase the chance of winning. Moreover, the hot hand belief can also be used for an allocation strategy if base rates are not known, such as in pick-up games.

Burns $(2001,2004)$ and Burns and Corpus (2004) suggested that believing in the hot hand may contribute to an adaptive behavioral strategy in basketball because it leads playmakers to pass the ball to a player with a higher scoring average in a game. Burns (2001) used a simulation of ball allocations to two virtual players and showed that behavior based on the hot hand belief resulted in higher average scores for the team than when the belief was ignored. Allocating the ball to the hot player would result in a small but important advantage of about one point in every seven or eight games. Furthermore, Burns argued that the greater the variability in the base rate of a player's scoring performance, the greater the advantage of the hot hand belief. Burns's mathematical model assumes that playmakers cannot detect base rates directly; their belief in the hot hand provides another, indirect source of information.

To determine whether the hot hand exists in volleyball and is used adaptively, we conducted two sets of studies. In Study 1A we

\footnotetext{
${ }^{1}$ Note that the gambler's fallacy, in contrast, predicts that a streak of events of the same class with stationary probabilities (e.g., "reds" in roulette) will more likely be followed by an event from a different class ("black"; Laplace, 1814/1951). It has been argued that this paradox can be explained by people's beliefs regarding the process that generates the specific sequence at hand (Ayton \& Fischer, 2004; Burns \& Corpus, 2004; Caruso \& Epley, 2004; Oskarsson et al., 2009). The hot hand belief is driven by people's assumption that the generating process is controlled by will (e.g., by athletes), whereas the gambler's fallacy assumes that the generating process is stochastic (e.g., in roulette).
} 
asked whether athletes believe in the hot hand and in Study 1B tested its existence in volleyball. In Study 2A we investigated how sensitive coaches can detect streaks, and in Study 2B we tested whether playmakers' allocation behavior is influenced by the belief. Volleyball is played six against six players: three players in the front row, who are allowed to spike over the net, and three players backcourt. One team serves the ball to the other side of the net. The next serve is given to the team that won the last rally (rotation rule). This rule requires players to rotate one position further when they serve after the opposing team's serve, meaning that players who play in front rotate to the back. After each set, teams change sides of the court. The team that wins three sets wins the game. The final score of the sets is 25 points ( 15 points in the event of a final fifth set) when one teams leads by two points; otherwise, the game is continued until one team leads by two points.

\section{Study 1A: Do Athletes Believe in the Hot Hand in Volleyball?}

Previous research argued that even if people believe in the hot hand in basketball, players do not necessarily possess a hot hand (e.g., Gilovich et al., 1985). The opposite result, that is, evidence for streakiness in players' performance, does not guarantee that a hot hand belief exists either. Therefore, we tested how strong the belief in the hot hand is in volleyball compared with in other sports and gambling. Previous research indicated that belief in the hot hand is context-specific, such that within the same person the belief varies between sport and nonsport situations (Caruso \& Epley, 2007; Tsyska, Zielonka, Dacey, \& Sawicki, 2007).

\section{Method}

\section{Participants}

Ninety-four sport science students (78 male, 16 female; mean age $24.2, S D=2.57$, mean sport experience, $M=12.8$ years, $S D=5.6)$ at the German Sport University Cologne participated in this study for partial course credit and provided written informed consent.

\section{Materials}

We developed a questionnaire based on previous studies of the hot hand belief in volleyball (Gula \& Köppen, 2009) that asked six questions. First, we listed all sports that were previously investigated in a review of the hot hand in sports (Bar-Eli et al., 2006) and added sports from recent studies not included in the review. The order of sports was randomized (archery, basketball, baseball, billiards, bowling, darts, golf, hockey, horseshoe pitching, soccer, table tennis, team handball, tennis, volleyball). Second, we piloted questions about roulette, in which the inversed hot hand belief, known as the gambler's fallacy, is found (Sundali \& Croson, 2006). Finally, we asked participants directly whether they believed in the hot hand in volleyball.

\section{Procedure}

Before receiving the questionnaire, participants were provided with a context-free definition of the hot hand: "Hot hand is defined as the higher probability in, for instance, sports to score again after two or more hits compared with two or three misses." They were then asked five questions: (i) Rank the following sports in the order in which you believe that the hot hand phenomenon is most present. (ii) Imagine that you are playing roulette. What color will occur after a sequence of two or three reds? (a) The probability of red is higher than black; (b) the probability of black is higher than red; (c) both are equally probable. (iii) Do you believe that a player should bet on red after a series of two or three reds? Yes/No. (iv) Do you believe in the hot hand in volleyball? Yes/No. (v) Do you believe that playmakers should play to a player that is hot? Yes/ No. In a final open question, they were asked to relate their individual sport experience by naming all sports they performed or had once performed at club level.

\section{Results and Discussion}

Eighty-six of the 94 athletes believed in the hot hand in volleyball (about 91\%). The average rank order of the 10 sports in which the hot hand was believed to occur was computed for all 94 athletes and was as follows (from most to least likely): basketball, volleyball, darts, billiards, bowling, baseball, golf, tennis, horseshoe pitching, and table tennis. We checked whether participants' individual experience in specific sports might influence belief for a particular sport, but this was not the case. The hot hand belief was distributed over the top 10 sports, independent of the sport in which an athlete had expertise. The majority of participants (about $81 \%$ ) selected (b) that "the probability of black is higher than red" after two or three reds in roulette.

This study provides first evidence that athletes believe in the hot hand in volleyball, which supports independent evidence from a study in our lab (Gula \& Köppen, 2009). However, does this belief reflect reality?

\section{Study 1B: Is There a Hot Hand in Volleyball?}

In Study 1B we tested whether a hot hand in fact exists in volleyball. We began with a brief discussion of how to detect a hot hand, a fiercely debated topic. How can the stability and existence of the hot hand belief be defined? Gilovich et al. (1985) stated that fans are right to believe in the hot hand if players' performance is either nonstationary or dependent. Based on athletes ' retrospective reports of experienced "hotness," Hales (1999) maintained that the belief is more appropriately tested by tests of stationarity than by tests of dependence. Both types of tests have the drawback that they rely on perfect runs of either hits or misses. In contrast, Larkey et al. (1989) argued that people may believe that a performance sequence reflects hotness, even if the sequence contains misses, which studies such as those of Gilovich et al. (1985) simply failed to detect. Thus, alternative tests should be developed that include imperfect sequences. However, for the study, we followed the "classical" analytical approach, assuming that if unusual variability in performance can be found, alternative tests are not necessary.

\section{Method}

We analyze the offensive performance (sequences of successive spikes) of male players in Germany's first-division volleyball 
league. Gilovich et al. (1985) discussed and rejected the hypothesis that the failure to detect the hot hand is a result of the opposing team's intensified defensive strategies against the hot player. In volleyball, however, allocation to a player cannot be hindered directly because the teams are separated by a net. ${ }^{2}$ And before the serve, the setter (playmaker) is not able to use many temporal or spatial cues from the opposing team for deciding to whom to pass the ball. Moreover, at first-division level, only one playmaker passes the ball to the final attacker. In these respects, volleyball provides a more controlled environment than basketball for the study of "streakiness" in players' performance data.

\section{Inclusion Criteria}

We evaluated the extent to which performance deviated from average within a short time frame (runs within a game) or long time frame (across games). The database we used (TopScorer) consists of 37,000 successful hits (spikes) and misses in the order of their occurrence in each game for more than 100 male players of 226 games in the German first-division volleyball league. In our analyses, we refer to miss and hit based on the TopScorer definitions, where a miss is an error committed when the attacker smashes the ball into the net or outside the playing field or steps over the attack line. A hit is a point for the attacking team by hitting the ball to the opponents' field such that the ball cannot be successfully defended. ${ }^{3}$ First-division volleyball teams, consisting of professionals or semiprofessionals, are the highest performance league in Germany, where there are no collegiate teams as, for instance, in the United States. For the present analysis, we used players from the best teams that entered the play-offs. Play-offs alone were used for calculating base rates and hotness, where the pressure to win is so high that only best performance matters, meaning that effective players will stay on the field and noneffective players will be substituted more quickly than in regular games. To check for individual streaks, one needs long sequences of hits and misses; therefore, only players who were constantly playingthat is, who attacked more than 40 balls in all play-off gameswere analyzed, which resulted in a total of 26 top players (see Table 2 for the distribution of individual players' attacks between 44 and 319 for all play-off games).

From these sequences, we tested the existence of random or nonrandom sequences using conditional probabilities and a runs test, exactly as in the Gilovich et al. (1985) study. In addition, we analyzed whether higher base rates result in significant lag 1 autocorrelations.

\section{Results}

\section{Probability of Scoring After Hits and Misses}

For the set of 26 offensive players we found base rates $\left(p_{\text {hit }}\right)$ ranging from .59 to .98 (unweighted $M=.9 ; S D=.08$ ) over the play-offs period (see Table 1). These high base rates in volleyball as compared with those in basketball led to a small number of sequences of misses. One reason is that we selected our sample from play-offs reflecting only the best players of the league. Another reason is that the definition of a miss in the database is restricted to spikes that do not go over the net or that land outside the field, meaning that neutral attacks in which the opposing team defends are not counted. Therefore, we could only test whether the probability of a hit is different after one miss or hit, following Gilovich et al.'s (1985) analyses.

Distributions of the conditional probabilities were analyzed across the 15 players for whom the total number of hits following a miss was equal or larger than 10 (column 5, Table 1). The unweighted mean probability of hitting conditioned on one miss was .67 $(S D=.21)$ and $.90(S D=.05)$ conditioned on one hit (one-sided $t(14)=4.29 ; p=.001$; Cohen's $d=1.1$ ). Weighting the probabilities by the ratio of the respective individual to the column total leads to similar results but with a reduced effect size (one-sided $t(14)=1.93 ; p=.037$; Cohen's $d=.5$ ). Whether these results provide evidence for cold or for hot streaks was explored by testing both conditional probabilities of a hit against the overall base rate. Effect sizes were higher for the difference between the overall base rate and the probability of a hit after a miss, $t(14)=$ 4.78; $p=.001$; Cohen's $d=1.2$; weighted by relative total: $t(14)=1.97 ; p=.035$; Cohen's $d=0.5$, than between the base rate and the probability of a hit after a hit, $t(14)=1.98 ; p=.034$; Cohen's $d=0.5$; weighted by relative total: $t(14)=1.53 ; p=$ .074 ; Cohen's $d=0.4$. This suggests that the streakiness resulted more from "coldness" than from hotness. As can be seen from Table 1, similar tests of the conditional probabilities of a hit after two or more misses are not feasible because the corresponding totals are too low. Instead, we assessed streakiness by testing whether the players' sequences contained fewer runs than expected.

\section{Runs Test}

We conducted a Wald-Wolfowitz runs test for all 26 players and used the same criterion as in Gilovich et al. (1985) for classifying a player as hot, which in our data results in a player's $Z$-value above 1.96. For this test, each sequence of consecutive hits or misses counts as a run. Thus, the more consecutive hits (or misses) a sequence contains, the fewer runs can be observed. In Table 2, the observed number of a player's runs is compared with the expected number of runs according to his base rate. Twelve of the 26 players had fewer runs than expected (bolded in Table 2); only one or two of the 12 would be accounted for by chance. The number of volleyball players with fewer runs than expected is substantially higher than among the basketball players studied by Gilovich et al. (1985) and Koehler and Conley (2003), where only one or two players, respectively, were found to have a significantly lower or higher number of runs than expected. The analysis of runs leads to the same conclusion as in the previous analysis of condi-

\footnotetext{
${ }^{2}$ It is important to note that the playmaker's ball allocation cannot be directly hindered as it can in open field sports such as basketball, team handball, or soccer, in which man-marking is a typical strategy to reduce allocation to one specific player. In volleyball, the defense can adjust to opponent players' behavior by organizing the block at the net or by positioning the backcourt defense players.

${ }^{3}$ In professional volleyball, one definition of a players' base rate is the overall probability of scoring, in which errors also include those spike attempts that were successfully blocked. Because the TopScorer database did not contain this information, the present analysis uses a base rate definition of a conditional probability of scoring given that the player was not blocked.
} 
Table 1

Analyses of Conditional Probabilities for the Performance of 26 Top Players in the German Male First-Division Volleyball League

\begin{tabular}{|c|c|c|c|c|c|c|c|c|c|c|}
\hline Player & $P_{\text {hit }}$ & Total & $P_{\text {hit }} / 1_{\text {miss }}$ & Total & $P_{\text {hit }} / 1_{\text {hit }}$ & Total & $P_{\text {hit }} / 2_{\text {hit }}$ & Total & $P_{\text {hit }} / 3_{\text {hit }}$ & Total \\
\hline 1 & .91 & 123 & .60 & 10 & .94 & 112 & .94 & 105 & .94 & 99 \\
\hline 2 & .98 & 176 & & 3 & .98 & 172 & .99 & 168 & .99 & 165 \\
\hline 3 & .95 & 186 & & 9 & .96 & 176 & .96 & 168 & .96 & 161 \\
\hline 4 & .94 & 319 & .94 & 17 & .94 & 301 & .96 & 284 & .96 & 272 \\
\hline 5 & .93 & 196 & .93 & 14 & .93 & 181 & .93 & 168 & .94 & 155 \\
\hline 6 & .93 & 308 & .67 & 21 & .95 & 286 & .96 & 271 & .97 & 259 \\
\hline 7 & .95 & 108 & & 4 & .95 & 103 & .95 & 98 & .95 & 92 \\
\hline 8 & .86 & 63 & & 9 & .87 & 53 & .87 & 46 & .90 & 40 \\
\hline 9 & .85 & 101 & .53 & 15 & .91 & 85 & .91 & 76 & .90 & 68 \\
\hline 10 & .95 & 143 & & 7 & .96 & 135 & .96 & 128 & .96 & 122 \\
\hline 11 & .89 & 108 & .91 & 11 & .89 & 96 & .88 & 85 & .92 & 75 \\
\hline 12 & .81 & 100 & .63 & 19 & .85 & 80 & .91 & 67 & .92 & 61 \\
\hline 13 & .89 & 161 & .49 & 17 & .88 & 143 & .90 & 125 & .92 & 112 \\
\hline 14 & .59 & 44 & .12 & 17 & .88 & 26 & .87 & 23 & .90 & 20 \\
\hline 15 & .95 & 75 & & 4 & .97 & 70 & .97 & 67 & .97 & 64 \\
\hline 16 & .73 & 79 & .62 & 21 & .77 & 57 & .79 & 43 & .82 & 33 \\
\hline 17 & .92 & 205 & .58 & 10 & .95 & 194 & .95 & 183 & .95 & 173 \\
\hline 18 & .85 & 149 & .83 & 23 & .85 & 125 & .87 & 105 & .88 & 90 \\
\hline 19 & .93 & 119 & & 8 & .95 & 110 & .96 & 104 & .96 & 99 \\
\hline 20 & .98 & 118 & & 2 & .98 & 114 & .98 & 111 & .98 & 108 \\
\hline 21 & .89 & 80 & & 8 & .92 & 71 & .91 & 64 & .93 & 57 \\
\hline 22 & .89 & 87 & .80 & 10 & .89 & 76 & .91 & 67 & .92 & 60 \\
\hline 23 & .97 & 197 & & 6 & .97 & 190 & .97 & 184 & .98 & 178 \\
\hline 24 & .92 & 198 & .75 & 16 & .93 & 181 & .94 & 168 & .96 & 157 \\
\hline 25 & .84 & 118 & .58 & 19 & .89 & 98 & .91 & 86 & .90 & 77 \\
\hline 26 & .92 & 117 & & 9 & .93 & 107 & .94 & 98 & .95 & 91 \\
\hline
\end{tabular}

Note. Sequences of hits and misses as probabilities after one miss $\left(\mathrm{p}_{\mathrm{Hit}} / 1_{\mathrm{Miss}}\right)$ or one $\left(\mathrm{p}_{\mathrm{Hit}} / 1_{\mathrm{Hit}}\right)$, two $\left(\mathrm{p}_{\mathrm{Hit}} / 2_{\mathrm{Hit}}\right)$, or three $\left(\mathrm{p}_{\mathrm{Hit}} / 3_{\mathrm{Hit}}\right)$ hits. Data are from season $1999 / 2000$. Total refers to the number of attacks; $p_{\text {hit }} / 1_{\text {miss }}$ is calculated only for totals $\geq 10$.

tional probabilities: In volleyball, a substantial number of players exhibit streakiness.

\section{Autocorrelations}

In a third test of the hot hand, we computed the autocorrelations as described above and found higher autocorrelations between successive hits in volleyball, compared with Gilovich et al.'s (1985) basketball data. The autocorrelation values (lag 1) for each player are shown in Table 2. We used lag1 values as indicators for a positive correlation as these measure the direct influence of the previous hit/miss to the next trial. Autocorrelations around zero $( \pm$ .1) are found for 14 of 26 of the top players; the remaining 12 players show significant autocorrelations between .1 and .7, indicating that successive attacks are dependent. Twelve players could be termed streaky according to both autocorrelation and the runs test. A similar result was not observed in the Gilovich et al. (1985) data, possibly because base rates were lower (around .5) and less variable. However, the only player with a base rate higher than 6 in their basketball study also received a significant but negative autocorrelation.

\section{Variability of Players' Individual Base Rates Between Sets and Games}

A key reason for playmakers to rely on the hot hand belief to make better allocations would be a systematic variability of players' performance between sets (up to five sets are played in a game of volleyball) and between games. For instance, local base rates ${ }^{4}$ in individual games within the regular season ranged from .45 to .70 for player A (no. 1 in Table 1) and from .54 to .86 for player B (no. 18 in Table 1). These players' variability was used as a guideline for a realistic manipulation of within-game sequences in the subsequent experiments (Studies $2 \mathrm{~A}$ and $2 \mathrm{~B}$ ). When looking at the within-game variability from set to set, player A showed a range of local base rates from .20 to .87 and player B from .33 to .90 . We found this large variability in more than half of the players, both within a game (i.e., between the maximum of five sets) and between the maximum of 15 games (see Table 2 base-rate column for significant variation indicated by an asterisk). This variability between sets and games is consistent with the findings in the previous sections that the hot hand exists in the data set and provides the precondition for an adaptive reliance on the hot hand.

\section{Correlations Between Number of Allocations and Players' Performance}

We correlated the number of playmakers' allocations to players and players' performance that may be indicative of the relation between both. First we correlated the observed number of runs and the number of allocations of a playmaker (Table 2, Total number of attacks). We found a correlation of .47 $(p=.008)$, which is about the same as a correlation of $.49(p=.005)$ between the base rate (Table $2, \mathrm{p}_{\text {hit }}$ ) and the number of allocations. Thus, both base

\footnotetext{
${ }^{4}$ We use the term local base rate when referring to the relative performance within a game, set, or half-set.
} 
Table 2

Analysis of Runs and Autocorrelation for the Performance of 26 Top Players in the German Male First-Division Volleyball League

\begin{tabular}{|c|c|c|c|c|c|c|c|c|}
\hline Player & Base rate $\left(\mathrm{p}_{\text {hit }}\right)$ & Number of attacks & Hits & Misses & Observed runs & Expected runs & $Z$ & Autocorrelation \\
\hline 1 & $.91^{*}$ & 123 & 112 & 11 & 14 & 21.0 & -3.98 & .31 \\
\hline 2 & .98 & 176 & 173 & 3 & 7 & 6.9 & .25 & -.02 \\
\hline 3 & .95 & 186 & 177 & 9 & 15 & 18.1 & -2.56 & .18 \\
\hline 4 & .94 & 319 & 301 & 18 & 34 & 35.0 & -.52 & .00 \\
\hline 5 & .93 & 196 & 182 & 14 & 26 & 27.0 & -.55 & .01 \\
\hline 6 & .93 & 308 & 287 & 21 & 29 & 40.1 & -5.05 & .28 \\
\hline 7 & $.95^{*}$ & 108 & 103 & 5 & 10 & 10.5 & -.62 & -.04 \\
\hline 8 & $.86^{*}$ & 63 & 54 & 9 & 15 & 16.4 & -.75 & .09 \\
\hline 9 & $.85^{*}$ & 101 & 86 & 15 & 17 & 26.5 & -3.81 & .37 \\
\hline 10 & $.95^{*}$ & 143 & 136 & 7 & 13 & 14.3 & -1.22 & .10 \\
\hline 11 & .89 & 108 & 96 & 12 & 22 & 22.3 & -.17 & -.02 \\
\hline 12 & .81 & 100 & 81 & 19 & 25 & 31.8 & -2.23 & .22 \\
\hline 13 & .89 & 161 & 144 & 17 & 35 & 31.4 & 1.52 & -.12 \\
\hline 14 & .59 & 44 & 26 & 18 & 6 & 22.3 & -4.98 & .74 \\
\hline 15 & .95 & 75 & 71 & 4 & 6 & 8.6 & -3.14 & .22 \\
\hline 16 & $.73^{*}$ & 79 & 58 & 21 & 27 & 31.8 & -1.41 & .15 \\
\hline 17 & $.95^{*}$ & 205 & 195 & 10 & 21 & 20.0 & .75 & -.05 \\
\hline 18 & $.85^{*}$ & 149 & 126 & 23 & 39 & 39.9 & -.29 & .02 \\
\hline 19 & $.93^{*}$ & 119 & 111 & 8 & 11 & 15.9 & -3.71 & .33 \\
\hline 20 & .98 & 118 & 115 & 3 & 5 & 6.8 & .22 & -.02 \\
\hline 21 & $.89^{*}$ & 80 & 72 & 8 & 15 & 15.4 & -1.14 & .13 \\
\hline 22 & .89 & 87 & 77 & 10 & 17 & 18.7 & -2.06 & .10 \\
\hline 23 & .97 & 197 & 191 & 6 & 11 & 12.6 & -2.06 & .14 \\
\hline 24 & $.92^{*}$ & 198 & 182 & 16 & 25 & 30.4 & -2.63 & .18 \\
\hline 25 & $.84^{*}$ & 118 & 99 & 19 & 23 & 32.9 & -3.41 & .31 \\
\hline 26 & $.92^{*}$ & 117 & 108 & 9 & 17 & 17.6 & -.41 & .04 \\
\hline
\end{tabular}

Note. Bolded numbers represent players with $Z$-values of $\geq 1.96$. Asterisk $\left(^{*}\right)$ in Column base rate ( $\mathrm{p}_{\text {hit }}$ ) indicates significant variation between sets and games. Data are from season 1999/2000. Z refers to the standardized test statistic, that is, the difference between the observed and the expected number of runs divided by the standard deviation of the expected number of runs. Autocorrelation scores reflect lag 1 autocorrelation.

rate and runs relate to allocation behavior. If we control for base rate in a partial correlation between observed runs and number of allocations, we find a correlation of .61 $(p=.001)$. Assuming that fewer observed runs than expected represent streakiness, we computed the degree of streakiness as the difference between the expected and observed number of runs. For the 12 streaky players in Table 2, we found a partial correlation of .66 ( $p=.026)$ between the degree of streakiness and the number of allocations (controlling for base rate). These results indicate that allocations are associated with runs and streakiness (hot hands) and thus playmakers' allocations cannot be explained solely by players' base rates.

\section{Discussion}

In our analysis of 26 top players, we found that (i) all three measures - conditional probabilities, runs, and autocorrelationprovided evidence of streakiness in about half of the players' hit-and-miss patterns and (ii) players' individual base rates varied between sets and games. This result differs from the stable patterns found in basketball by Gilovich et al. (1985) and Koehler and Conley (2003). One possible structural explanation for the difference between the studies in volleyball and basketball lies in the nature of each sport. If there is a hot hand in basketball, it may not be detected in the hit-and-miss data, because opponent players can react by using counterstrategies against the hot player. This is less possible in volleyball, where a net separates opposing teams. However, even when defense was absent in basketball, that is, when players had free throws (Gilovich et al., 1985) or in threepoint shootout contests (Koehler \& Conley, 2003), hot hand was not found either. Whether the hot hand belief is restricted to basketball or can be generalized to other sports or other domains (see Gilovich, 1993; Gilovich, 1985; Tversky \& Gilovich, 1989) is still under debate, but a recent review concluded that the hot hand should be more present in sports or situations within a sport in which defense can be eliminated or limited (Bar-Eli et al., 2006).

Study 1 provides the first evidence of streakiness in offensive performance in volleyball. There are two possible limitations to this study. First, the autocorrelations were calculated throughout the full sequence of hits and misses; therefore, the last hit or miss in play-off game 1 was compared with the first attack in play-off game 2. However, because there are only three to five play-off games in which an individual player can participate, this effect does not influence the results. Alternatively, running separate autocorrelations for each player for each game would potentially inflate alpha errors. A second limit is that defense pressure can change between games even with the same opponent in play-offs and therefore could inflate the streakiness in the data. Although we chose play-offs of the top-teams to limit this specific effect, the variability within a game and between games suggests that there is variability that could be perceived by coaches or fans, and could also alter playmakers' allocation strategies.

The current data suggest that in volleyball there is both streakiness ("hot hand") for half of the players and no streakiness for the other half. Thus, the hot hand belief reflects reality for half of the 
players but not for everyone. It is therefore necessary to show that coaches and playmakers can actually detect the subtle but systematic changes in players' performances beyond evidence of correlations. Study 2 addressed how sensitive coaches can perceive changes in performance (Study 2A) and how playmakers use the hot hand belief in their allocation decisions (Study 2B).

\section{Study 2A: Are Coaches Able to Detect Players' Changes of Performance Over Time?}

In Study 2A we evaluate how sensitive coaches are in detecting changes of performance and whether they used these to instruct playmakers how to allocate the ball. Sensitivity was measured for local changes of the average base rate of a player. A local base rate needs to be specified with respect to a reference class; here we consider one natural unit, the set in a volleyball game (e.g., six of 10 hits in the first set is better than two of 12 in the second). Although there is evidence that people are sensitive to changes in base rates (e.g., Oskarsson et al., 2009; Koehler, 1996 for overviews) nothing is known in the context of sports. For instance, a general finding is that within-subject variation in the base rate draws attention to the base rate (Gigerenzer, Hell, \& Blank, 1988; Mueser, Cowan, \& Mueser, 1999), but it is not known how accurately changes in the base rate of an individual player can be detected. Furthermore, Castaneda and Rodrigo (1998) showed that people are more sensitive to perceptual information than to information presented semantically (as is used in most experiments) when estimating the base rates of events. Because Castaneda and Rodrigo used stable nonspecific visual information, but in a real game coaches react to variable sport-specific stimuli, it is unclear how their result applies to sports. We therefore conducted an experiment that evaluated the sensitivity to picking up base rates of hits in dynamic and visually presented volleyball attacks.

\section{Method}

\section{Participants}

Sixteen coaches from Berlin with a B- or C-level coaching license in volleyball and a mean age of 37.5 years $(S D=10.3)$ participated in the study. In Germany, B-level coaches are allowed to coach up to the third highest and C-level coaches up to the second highest professional league level. They received 10 euros for participating. All coaches gave written informed consent beforehand.

\section{Apparatus}

The stimuli were video files showing the attacks of the two teams in a volleyball game. These were projected onto a large screen $(1.5 \times 2 \mathrm{~m})$ to ensure a realistic perceptual set-up. Video presentations are typically used by coaches and athletes to evaluate performance after a game (Lyons, 2003; Paiement, Baudin, \& Boucher, 1993). In the videos, we limited the attacks to sequences of two volleyball players, arranged so that player A always attacked from the left side of the display and player B always attacked from the right. A computer program randomly displayed either the left or the right player, who then performed either a hit (defined as a spike into the opponent's field) or a miss (a spike that sent the ball either outside the field or into the net).

\section{Task}

The task was to watch a game, which consisted of four sets of 44 attacks (176 attacks in total). Coaches were asked to watch the distribution of the setters' passes to two attacking players (as they would in a real game) and after the middle of each set (time-out). At the end of each set they were asked to write down instructions for the setters for allocating the ball to the two players as well as to recall the observed players' performances.

Coaches were given written instructions that they were required to analyze a playmaker's allocation strategy on a video. They did not know how the two attackers would perform beforehand. Before the experiment began, they viewed two scenes with each player (one hit, one miss) to familiarize themselves with the task. Subsequently, they were asked to (i) watch attacks by players A and B in a random pattern of sequences of 11 attacks by each player, (ii) after each sequence of 22 attacks, write down the distribution of balls to player A and to player B for the next 10 allocation decisions, and (iii) recall the performances of the two observed players (how many out of the last 11 attacks did player A-or B-hit?).

At the end of the experiment we distributed a questionnaire about the coaches' hot hand belief that contained the same items as in Gilovich et al. (1985) but adjusted to volleyball. We asked: (i) Does a player have a better chance of making a successful strike after having made two or three previous spikes than he does after having missed the previous ones? (ii) Consider a hypothetical player who has an average spike hit rate of $50 \%$. Please estimate (from $0 \%$ to $100 \%$ ) the player's average hit percentage after just having successfully spiked before __ and after just having missed the last spike __. (iii) Do you believe that playmakers play to a player that is hot? Yes/No.

\section{Material}

The sequences of players were distributed as ecologically validly as possible by matching sequences of successful hits and misses found for two players in the TopScorer database (Study 1B). The Institute for Applied Training Sciences (IAT) in Leipzig, Germany, provided us with audiovisual (AV) files of these players' successful hits and misses from both sides of the court, making it possible to manipulate the sets (after each set, teams change court sides) accordingly. The base rates used, the sequence of successful hits and misses, and the number of hits an average player attempted before the next side-out attacker rotated from the back row to the front in order to hit near the net (based on the rotation rule) were organized to reflect real-life game situations.

The AV files of the two players were edited in the following way. One team served and a player passed the ball to the playmaker, who then set the ball to the left outside position where a player either hit or missed. This player who hit was either player A (when shown on the left) or player B (when shown on the right).

Four sets of stimuli were prepared such that each set ended when a team reached 25 points, as in a real game. Each set consisted of 44 attacks ( 22 by each player). After half of the set (22 attacks, 11 for each player), a time-out was displayed. The base 
rates for the players in each half-set (22 points) were either the same (twice) or different (six times). The difference between the players' number of hits was varied from a one-point difference to a five-point difference to see how easily the base rates and differences between players could be picked up and used when defining allocation strategies for the playmakers. To avoid any potential ambiguities (e.g., a ball that was very close to an offside line), the score was displayed after each point.

\section{Results}

$92.3 \%$ of the coaches believed that a player has a better chance of making a successful strike after having made two or three previous spikes than he does after having missed the previous ones, indicating a general belief in the hot hand. Coaches' estimation of a player's hitting probability just after having made a strike was $55.5 \%$; their average estimation of a player's strikes just after having missed a strike was $46.8 \%$. This difference is smaller than Gilovich's results for basketball (61\% to $42 \%)$. $84.6 \%$ of the coaches believed that playmakers play the ball in accordance with this belief, indicating that coaches consider more factors for allocation than merely the hit rate of a player.

On average, the 16 coaches wrongly estimated the number of hits in the preceding half-set by only 1.2 balls out of 22 . License class, age, and gender did not influence the coaches' level of performance. Player A was evaluated as a slightly better hitter (by about 3 points), even though the mean base rates were the same over the game. The correlation between the estimated base rate of a player (how many hits of the last 11) and the allocation strategy (how many balls to player A and to player B) was $r=.55, p=$ .002 , over the entire game. This result indicates that the coaches were sensitive to changes in base rate and corrected their allocation strategies according to their base rate judgments.

\section{Discussion}

The results of the experiment indicate that coaches are sensitive to base rate changes and use them as cues for allocation decisions. This conclusion is supported by coaches' allocation instructions that correlated with the perceived base rate changes within the two players. The observed number of hits influenced the coaches' instructions for allocating the next 10 attacks. All volleyball coaches' base rate sensitivity was highly accurate, with an average error of about 1 hit or miss for each player out of 22 attacks. In general, coaches believe in the hot hand and also say that their playmakers use the hot hand belief for their allocations. From the coaches' allocation instructions, it is evident that they would not play all balls to the player with the momentarily higher performance because of potential side effects, such as a specific player's fatigue.

The changes in coaches" allocation instructions show that they go beyond using the overall base rate of players and take shortterm performances into account, which in the present study would result in equal distribution to both players. In the next step, we tested whether players actually allocate balls in an adaptive way depending on the perceived "hotness" of players and base rate. For Study 2B, we therefore used the same paradigm as in Study 2A and asked players to allocate balls to two players who have either the same or a different base rate and who vary in their degree of being hot.

\section{Study 2B: How Do Playmakers Use the Belief for Allocation decisions?}

\section{Method}

\section{Participants}

Twenty-one persons (11 male, 10 female; age: $M=23.5$ years, $S D=2.9$ ) with volleyball experience took part in the experiment. Volleyball experience was defined as participating in a major course in volleyball that requires playing at adult club level for at least one year. All participants were students in physical education or sport science. Participants played on average at regional level (from third lowest level to third highest national level) and thus below the professional first and second league. None of them had taken part in Study 2A. They received 10 euros for their participation and could win another 20 euros based on their performance. All gave written informed consent before participating in the experiment.

\section{Apparatus and Material}

The task was again presented as a digital video test on a computer, projected at a presentation size of $1.5 \times 2 \mathrm{~m}$ at a distance of $3 \mathrm{~m}$ from the participant. The software $(\mathrm{C}++$, Czienskowski, 2002) displayed AV files and collected data from the participants. In this study the participants' task was to allocate the ball to one of two players before every attack, define their allocation strategy in advance, and estimate the two players' base rates. Note that in the present context the term base rate is used to refer to the relative frequency of hits of a player per half-set of the game.

The same AV files as in Study 2A were used, but the order of presentation was varied. The manipulated base rate changes were-according to our analysis of the TopScorer Database-a typical distribution of players' real base rate changes.

Four conditions counterbalanced in order were designed (see Table 3): (i) base rates are equal for both players and both show a pseudorandom distribution of hits and misses (no hot hand); (ii) base rates are equal for both players but one player is hot; (iii) base rates are different and both players demonstrate a pseudorandom distribution of hits and misses; and (iv) base rates are different and one player is hot. We varied the base rates in both the "same base rate" and "different base rate" conditions to model real base rate changes between sets (see Table 3). A pseudorandom distribution was defined as no more than two hits or two misses in a row for each player. In the hot hand condition, a sequence of three hits occurred at least twice. The number of attacks (hits and misses) was arranged based on the distributions found in the database of real competitions. We restricted the hot hand sequences so that no more than four spikes in a row were made by one player.

To determine experimentally whether belief in the hot hand influences behavior, we constructed series of hits and misses that were either pseudorandom (no more than two consecutive hits or misses) or "nearly hot" for each player and half-set (three to four consecutive hits). When constructing the pseudorandom condition we used a positive $Z$-value between 0 and 1 as a criterion, reflect- 
Table 3

Tests Consisted of Four Sets (Eight Half-Sets With 22 Trials/Attacks Each) in Which Base Rates of Player A and Player B Were the Same (1, 2, 7, 8) or Different (3-6), and Either One Player Was Hot ("Hot: Yes") and the Other Player had a Pseudorandom Sequence ("Hot: No") or Both Had Pseudorandom Sequences

\begin{tabular}{|c|c|c|c|c|c|c|c|c|c|}
\hline Half-set & & 1 & 2 & 3 & 4 & 5 & 6 & 7 & 8 \\
\hline \multirow[t]{2}{*}{ Player A } & $\mathrm{BR}$ & .50 & .64 & .40 & .73 & .45 & .67 & .50 & 64 \\
\hline & Hot & No & Yes & No & No & No & Yes & No & No \\
\hline Allocation (mean, $S D$ ) & & $12.4(3.5)$ & $12.3(3.1)$ & $10.2(3.1)$ & $10.0(2.0)$ & $12.1(3.2)$ & $13.3(3.7)$ & $12.4(3.6)$ & $9.5(3.8)$ \\
\hline Estimation (mean, $S D$ ) & & $52.38(13.4)$ & $59.74(13.01)$ & $40.69(11.36)$ & $52.81(15.64)$ & $60.17(17.59)$ & $61.03(16.55)$ & $48.05(13.21)$ & $50.21(8.43)$ \\
\hline \multirow[t]{2}{*}{ Player B } & $\mathrm{BR}$ & .50 & .64 & .58 & .55 & .73 & .40 & .50 & .64 \\
\hline & Hot & No & No & No & Yes & No & No & No & Yes \\
\hline Allocation (mean, $S D$ ) & & $9.6(3.5)$ & $9.7(3.1)$ & $11.8(3.1)$ & $12.0(2.0)$ & $11.9(3.1)$ & $8.7(3.6)$ & $9.6(3.6)$ & $12.5(3.8)$ \\
\hline Estimation (mean, $S D$ ) & & $47.6(11.46)$ & $43.72(13.05)$ & $58.44(9.77)$ & $56.71(13.45)$ & $41.99(15.34)$ & $43.28(15.72)$ & $45.88(10.93)$ & $64.5(10.32)$ \\
\hline Condition & & 1 & 2 & 3 & 4 & 3 & 4 & 1 & 2 \\
\hline
\end{tabular}

Note. $\quad \mathrm{BR}=$ base rate; Hot $=$ hot hand; Allocation $=$ mean number of allocations to the respective player $(\mathrm{SD}) ;$ Estimation $=$ estimation of local bases rates. Conditions are explained in the text.

ing slightly more or exactly the same number of actual runs as expected given the players' base rates. In the hot condition we deliberately introduced runs ("nearly hot") that would not satisfy the statistical definition of a hot hand streak $(Z>1.96$, see Study 1B) yet were long enough for participants to associate them with an increased probability of a hit. We assumed that if participants estimated the probability of a hit to be higher after two or three hits than after two or three misses (the hot hand question in Study 1A), then runs of length three and four should be perceived as streaky. The goal was to examine the effect of perceived hotness, as opposed to actual streaks, on allocation decisions.

In comparison, in the original study of the hot hand phenomenon (Gilovich et al., 1985), the nine players of the Philadelphia 76ers had a mean number of runs of about 215 and an expected number of runs of 210, which results in a small negative $Z$-value of -0.56 . Daryl Dawkins had the highest negative $Z$-value of -3.09 , representing an expected number of runs of about 190 and an observed number of runs of 220 .

We controlled for the influence of a specific player to ensure that both players were hot equally often and had the same base rate over the entire game. In half-sets 1 and 7 (Condition 1) base rates were the same for both players, and their hits and misses were distributed pseudorandomly. Neither cue (differences in base rates or streaks) in this control condition provided any information that might be exploited for an allocation strategy other than random. In half-sets 2 and 8 (Condition 2) the base rates were also the same but the sequence of spikes and misses was manipulated systematically to be hot ( 3 to 4 hits in a row) for one player and pseudorandom for the other.

Because there is no difference in performance between the players with equal base rates, only the hot hand can serve as an allocation cue in these conditions. This environmental structure resembles a typical "pick-up" game situation, where base rates cannot be used as an allocation cue (given that they are not known) and streaks may instead serve as cues to infer the underlying level of performance and influence allocation. In the other half-sets, the players' base rates were varied.

In half-sets 3 and 5 (Condition 3 ) the sequences of successful spikes and misses were pseudorandom for both players; hence, any consistent allocation behavior of participants can be attributed only to the differences in the players' base rates. Note that Conditions 2 and 3 were especially designed to determine whether the two kinds of information provided (i.e., sequence structure and base rates) independently influence allocation behavior.

In Condition 4 the effect of both cues on allocation behavior was investigated. First, the sequence in half-set 6 was manipulated to be hot for the player with the higher base rate. Then the sequence in half-set 4 was manipulated to be hot for the player with the lower base rate.

\section{Procedure}

Participants were individually tested in a session that took about $60 \mathrm{~min}$. At the beginning of the session, the experimenter presented a written introduction to the experiment and explained the performance-based payment system. Participants could then warm up for the test by observing 10 pilot video clips and pressing the button corresponding to the player to whom they wanted to allocate the next ball. Four sets of a volleyball game were presented. Each set consisted of 44 attacks (balls in play until the final score, with one team winning 25 points). After the first 22 attacks (the time-out) participants were asked to type in a displayed text box on the screen how they wanted to continue their ball allocations to the players. Specifically, they were asked how many of the next 10 balls they wanted to distribute to player A and how many they wanted to distribute to player B.

After participants made their allocations for the following halfset, a new query appeared on the display, asking them to estimate for player A and player B how many of their 11 attacks in the preceding half-set were successful, that is, were hits. The final 22 attacks for the first set were presented when participants finished their base rate estimates. After the first set, the questions about their allocation strategy and base rate estimates were repeated. This procedure was repeated for all sets within the experiment. After the final set, participants were asked to fill in a questionnaire containing items on personal data and a section on their decision strategy, in which they could rank possible factors (base rate and structure of sequence) that influenced their decisions. 
In addition, the questionnaire included items regarding participants' belief in the hot hand. For the German participants, we translated the term hot hand literally, saying that someone is hot ("ist heiß"). In particular, the questionnaire explicitly asked about the use of the structure of the sequence and of the base rates, and to whom they would allocate the ball if, for instance, toward the end of the game the player with the lower base rate hit two or three balls in a row.

\section{Results}

\section{Hot Hand Belief Questionnaire}

As in Study 2A, two questions were asked regarding belief in the hot hand. These were followed by three additional questions concerning the information that had been manipulated in the experiment. First, we asked if it was important to allocate the ball to the player who had just hit two or three times. Nineteen of 21 participants (about 90\%) answered in the affirmative. Second, we asked if a player who had just hit the last two or three times had a better chance of hitting the next ball than a player who had just missed the last two or three balls. Fifteen of 21 participants (about $71 \%$ ) said yes. These results support the rationale behind the constructed sequences that runs of three and four hits would be perceived as hot by the majority of participants. When asked the third question, to rank the importance of different factors that influenced their decisions, participants ranked the players' hot hand as most important, followed by the "cold hand" (number of misses in a row), and the overall base rate in the set. Participants also stated that they used the hot hand or cold hand of the players to allocate their passes in seven of 10 decisions. When asked explicitly about a situation where one player has the higher base rate and the other has just made two or three hits but has a lower base rate, $62 \%$ of the participants claimed that they would allocate the ball to the latter player.

\section{Allocation Behavior When Base Rate and Hot Hand Information Are Present}

We found that participants (setters in the experiment) were sensitive to both the hot hand and the players' base rates, as indicated by their real allocation behavior. The hot hand cue was stronger than base rates in determining participants' allocation. That is, most participants relied on the hot hand consistently (see Figures 1, 2, and 3).

Condition 1. In this control condition, the players' base rates were equal and the sequences for both players were pseudorandom. The allocation to player A or player B was close to equal, with a nonsignificant tendency to pass more often to player A (see Table 3). Similarly, participants' estimates of the number of hits did not differ from the actual base rates in the videos.

Condition 2. We then considered the critical case in which the base rates of hits for the two players were the same but one of the players was hot. If participants used base rates alone, allocations to both players should be equal. Yet as shown in Figure 1, participants allocated balls more often to the hot player, even though the base rates were the same. This result holds regardless of whether player A or B was hot ( $t$ test player hot/not hot, $\eta^{2}=$ $.24 ; p=.02)$.

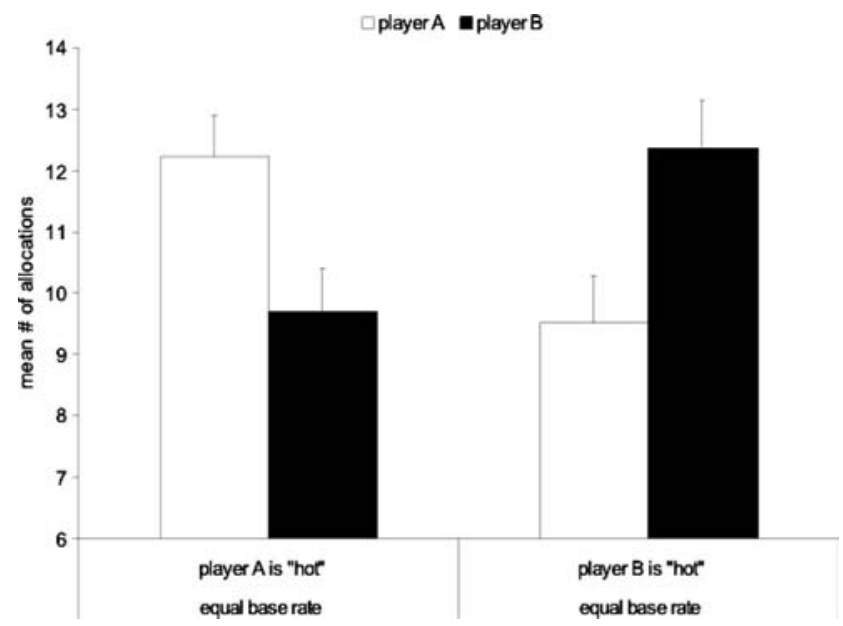

Figure 1. Mean number of allocations of the ball to player A and player $\mathrm{B}$ when base rates were equal but a hot hand existed for player A (left) or player B (right). Error bars represent standard errors.

As in Condition 1, the base rate estimates for the player with a hot hand were almost perfect. However, for the players with pseudorandom sequences, base rates were underestimated by about two more misses when player A was hot ( $t$ test real vs. estimated base rate, $\left.\eta^{2}=.34 ; p=.01\right)$ and similarly, when player B was hot $\left(\eta^{2}=.63 ; p=.001\right)$. This is a surprising finding, where a hot player appears to lead to a devaluation of other players (see General Discussion).

Condition 3. In this condition, one player had a higher base rate but neither player was hot. The question was whether participants allocate balls in a way that is sensitive to base rate differences when there is no hot hand. Figure 2 shows that allocations increased in both situations from about 10 to 12 ( $t$ test player with higher vs. lower base rate, $\eta^{2}=.16 ; p=.07$ ).

Condition 4. Here, participants were shown videos of players who had different base rates, in which one player was hot. The hot player had either the higher or the lower base rate. When player B had a hot hand but player A had the higher base rate (left side of Figure 3), more allocations were made to the hot player. The same result was found when player A had a hot hand and player B had the higher base rate $\left(t\right.$ test hot hand vs. higher base rate, $\eta^{2}=.42$; $p=.01)$. When the player with the hot hand also had the higher base rate (right side of Figure 3), the allocation to this player was higher ( $t$ test hot hand and higher base rate vs. no hot hand and lower base rate, $\left.\eta^{2}=.15, p=.03\right)$. In this situation, more allocations were made than in Conditions 2 and 3, where only hot hand or base rate was available (ANOVA, $\eta^{2}=.35 ; p=.01$ ).

An analysis of the base rate estimates showed the same surprising result as in Condition 2 but extended it to the case where base rates differed. If the player was hot and had the higher base rate, the participants estimated his base rate almost perfectly but underestimated the base rate of the other player by about two hits ( $t$ test estimated base rate for player A vs. player $\left.\mathrm{B}, \eta^{2}=.41 ; p=.001\right)$

\section{Do Players React in an Adaptive Way to Local Base Rate Changes?}

Because each participant was exposed to the four conditions, all of them were confronted with changing base rates, enabling us to 
test whether they could allocate balls in an adaptive way when a player's base rate suddenly changed. After viewing 22 attacks of player A and B in Half-Set 1, they were asked for estimates of both players' base rates (they were never asked whether a player was hot) and then asked how they would allocate the balls for the next half-set. Yet unknown to them at that point, the base rate in the next half-set changed for both players from .50 to .64. Whereas player A and player B had the same base rate in the first two half-sets, in Half-Set 3 player B had a higher base rate than player A. If participants reacted to this change in an adaptive way, the announced allocation should not be identical with the actual allocation in the following half-set but change in the direction of the new base rate. We analyzed the number of adaptive changes compared with nonadaptive behavior. An adaptive change is present, for instance, if an intended allocation prefers player A to B but in the following half-set, where player B has a higher base rate, participants abandon their intended allocation to player $\mathrm{A}$ and allocate more balls to player B. An adaptive change is also present if intended allocation is equal to both players because their base rates in the present set are equal, but in the next half-set, where player A has a higher base rate, participants allocate more balls to player A. A nonadaptive allocation strategy is present if participants remain loyal to their intended allocation, such as allocating more balls to player A in the next half-set, even if player B now has a higher base rate. Because there are eight half-sets, we can observe seven changes of base rates for player A/player $\mathrm{B}$. We found that, averaged over participants, an adaptation in the direction of the new base rates was present in five out of these seven cases. The correct changes from the intended allocations in response to the new base rates were on average one or two balls in the correct direction, reflecting base rate changes between half-sets of about .1 or .2. In the two cases in which intended allocation was not in the direction of the actual base rates, all half-sets had one player with a "nearly hot hand." Thus, the intended future allocations always followed the base rates significantly (all $p \mathrm{~s}<.05$ ), except in the two cases where the base rate conflicted with being

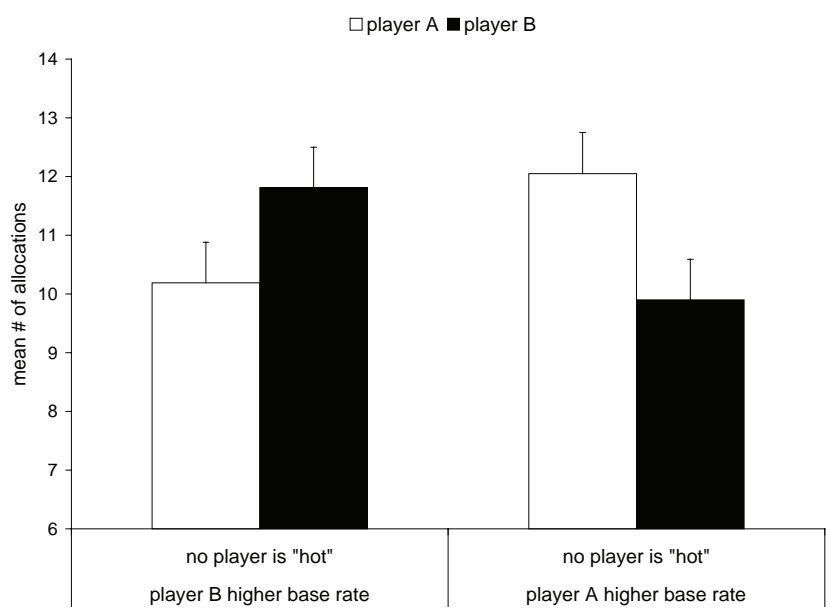

Figure 2. Mean number of allocations of the ball to player A and to player B when no hot hand existed for the players but the base rate for player B (left) or player A (right) was higher. Error bars represent standard errors.

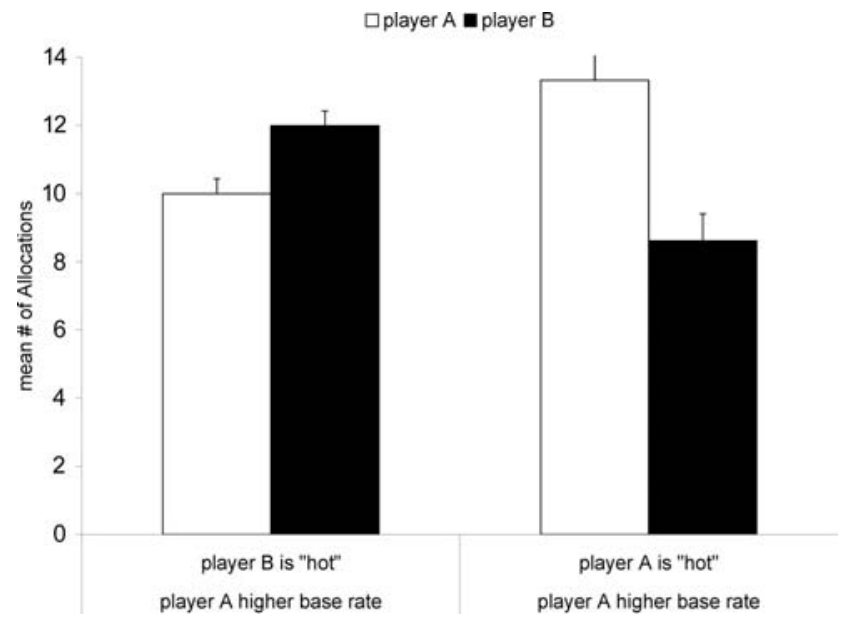

Figure 3. Mean number of allocations of the ball to player A and to player B when a hot hand and the higher base rate were present in the same player (right) or not (left). Error bars represent standard errors.

hot. For these cases, where the hot hand dominated, using the hot hand belief can be labeled nonadaptive.

\section{Discussion}

Study 2B showed that participants were able to detect a hot hand and used this information for their allocation decisions. Moreover, the hot player was allocated more balls even if his base rate was equal to or lower than that of the other player's. Whereas the hot hand dominated allocation decisions, comparison of the conditions showed that base rates alter the allocation frequencies. An unexpected result was that if one player was hot, the other player's base rate was systematically underestimated. This effect could be explained by increased attention to the hot player and more cognitive resources allocated to monitoring his performance. The trials results suggest that strategies based on few preceding events may perform better than those based on complete information about attackers' performance.

\section{General Discussion}

The previous studies addressed three questions: Do athletes and coaches believe in a hot hand in volleyball? Does the hot hand exist in volleyball? And is the hot hand belief used to inform allocation decisions? The results indicate that the answer is "yes" for all three questions. In our samples, $91 \%$ of 94 athletes (Study 1A), $90 \%$ of 21 athletes (Study 2B), and $92 \%$ of 16 coaches (Study $2 \mathrm{~A})$ believe in the hot hand. Depending on the criterion used, $53 \%$ of the 26 German top players show significant streaks, and $46 \%$ show significant autocorrelations (Study 1B). The key evidence that suggests an adaptive use of the hot hand belief is that playmakers allocate the ball more often to players with streaks and that this leads to better performance than when allocating the ball to the player with the higher average base rate (Study 2B).

In what follows, we discuss several open questions resulting from the present and previous research. 


\section{How Is the Hot Hand Belief Used in Real Allocation Decisions?}

Study 2B demonstrated that the hot hand of a player can directly influence allocation strategy. As shown in Study 2A, coaches use information on streaks to determine behavioral strategies, that is, allocation, and they believe that other professionals do the same. Study 1B showed that their belief in the hot hand is partly correct.

Moreover, information on streaks is not used merely because players' base rates or changes in their base rates cannot be directly detected (Burns, 2004); rather, the hot hand of players is used as information in addition to their base rates. Study 1B was the first to show correlations between number of playmakers' allocations and observed runs as well as correlations between allocations and base rates. As of yet we are not able to differentiate the information that influences allocation.

It is important to distinguish between hot hand defined as a performance difference within a player (the classical definition) and as a performance difference between players. The latter is the important information for allocation in the real game. This interpretation is supported by the results of Study $2 \mathrm{~B}$, in which the estimation of the two players' base rates was influenced nonequally such that the base rates were underestimated if the player was not hot. To explain this striking and potentially costly result, further investigation is necessary (Gilovich et al., 1985). For instance it could be assumed that a scout watching a game focuses mainly on absolute performance of an individual player, whereas the playmaker of a team as tested in this set of studies may rely on relative performance for his decisions.

A model for allocations in sports that goes beyond the models using streaks and base rate explored in Study 2B should incorporate further cues that players might use to allocate a ball. In the present research, we considered only the influence of players' base rates and hot hand on playmakers' decisions. Drawing on the studies conducted so far, we can begin to extract process details of these decisions. However, the possible mechanisms that a playmaker uses to allocate balls in volleyball and potentially in other sports are still far from being understood, and one step toward answering this question is taken by Köppen and Raab (in press). Another issue highlighted by Study 2B is that streaks influence the allocations more strongly when base rate differences between players are small. Finally, because the current manuscript focuses on positive streaks, it is not yet shown whether beliefs about the cold hand in sports result in fewer allocations to the player in question and in earlier substitution of that player in the game (but see Köppen \& Raab, in press).

\section{When Is the Use of the Belief in the Hot Hand Adaptive?}

Our key argument is that streaks within a small time unit of a set of a game exist and when these are picked up by the playmaker, better performance can result. By better we mean better than a random allocation strategy or a base-rate strategy. The results of Study 2B indicate that the belief in the hot hand is used when base rates change within a player across sets and between players within a set.

However, a hot-hand-only allocation strategy that ignores base rates does not appear foolproof. It can decrease performance if the hot player's base rate is lower than those of the other players. For instance, Figure 1 suggests that the effect of the hot hand allocation strategy is about two to three balls (of 22) more for the hot player. The strategy will lead to nonadaptive allocations if the base rate differences between the hot and the not-hot player compensate for this effect. This can only happen if the hot player has the lower base rate and the base rate difference results in three or more balls. In Study 2 b, Condition 4, we created such a situation, and playmakers continued to apply the hot hand strategy (if a hot player exists, allocate to him) even though it led to worse outcomes. In this condition, the base rates differed from .55 to .73 , that is, by 18 percentage points.

It remains unclear how often the combination of a hot hand with a substantially lower base rate occurs within a real volleyball team. In our data we have exactly one player (player 14, Table 2) who has a base rate of .59 (we used .55 in Study 2B, Condition 4) and a hot hand indicated by a high autocorrelation and $Z$ score. Let us consider this player in a team of six volleyball players on the court, of whom one is a playmaker and one is a defense player who does not attack, resulting in a choice to allocate the ball to one of four players. If the playmaker chose to allocate the ball to the hot player with the lower base rate systematically more often than reflected by his low base rate, this allocation would be ineffective. However, this can only occur if the other players are not hot and the hot player is on the court. Note that players with a lower base rate do not play often (e.g., player 14 played the lowest number, 44 attacks from a total of 3,804, compared with the highest number, 319 attacks, by player 4). Furthermore, in our database only one such player exists in one team of the final four teams of the play-offs, meaning that this situation is rare.

Based on autocorrelations and analyses of runs tests we know that players (at least in volleyball) have high base rates and that nonrandom sequences exist. We also know that coaches and playmakers are able to detect base rates and changes in base rates of players accurately in the simple and fairly realistic setting of our experiment. However, we do not know what playmakers more likely believe: (a) that a player is hot if he overshoots a specific level of base rates or (b) that a player is hot if a difference between players' base rates under monitoring meets a threshold. These issues deserve further experimental investigation to understand the important relation between the gradual increase or decrease of the hot hand belief during changes in situations, base rates, and individual behavior. Burns (2004) cited unpublished data that suggest that people are more likely to follow streaks (in our experiment, allocate balls to a hot hand player) in situations in which they perceive that options may differ in their probabilities of success. In turn, these are exactly the conditions under which following streaks is most adaptive, implying that people are sensitive in some way to the implications of the hot hand phenomenon.

The behavioral results of Study $2 \mathrm{~b}$ suggest that both cues (hot hand and base rate) are independently used for allocation strategy. These results extend the work of Burns (2004), who excluded equal base rates of the two players in his simulation. For instance, Study 2B showed that even if players had equal base rates, participants used the hot hand cue and passed the ball more often to the player with streaks. Participants were clearly following streaks. Following streaks when both players' base rates are equal is not 
maladaptive per se. If the defense cannot detect or exploit the allocation behavior, following streaks has at the very least a neutral impact on performance.

\section{What Do We Learn From Analyzing Streaks?}

In this article we showed that the hot hand as well as the belief in it exists in volleyball. One may ask why this result is of any importance. Yet every day millions of people watch, participate in, or report on sports events. Research on the hot hand belief and hot hand behavior has the potential to connect sports fans and coaches with psychological research and to apply experimental studies to a topic that has great meaning to many across the world. Moreover, beliefs about short-term sequential dependencies are also formed outside sports. Many if not most natural events (e.g., the daily weather) and human behaviors (e.g., parents' interaction with their children) are characterized by naturally occurring sequential dependencies.

The potential reasons for streak detection can be phylogenetic, as shown by Wilke and Barrett's evolutionary approach (2009), where the authors concluded that people have an inborn competence for detecting and using streaks based on a cognitive adaptation to the environment. Streak detection may be a result of experience, in which momentum and contrarian strategies are used based on experience or as a result of human learning, as shown by Carlson and Shu (2007; see Oskarsson et al., 2009 for an overview). The motivation and ability to detect streaks can help us beyond the domain of ball allocations, from making careful scientific observations to avoiding blunt superstition.

\section{References}

Adams, R. M. (1995). Momentum in the performance of professional tournament pocket billiards players. International Journal of Sport Psychology, 26, 580-587.

Ayton, P., \& Fischer, I. (2004). The hot hand fallacy and the gambler's fallacy: Two faces of subjective randomness? Memory \& Cognition, 32, 1369-1378. doi:10.3758/BF03206327

Bar-Eli, M., Avugos, S., \& Raab, M. (2006). Twenty years of "hot hand" research. The hot hand phenomenon: Review and critique. Psychology, Sport and Exercise, 7, 525-553. doi:10.1016/j.psychsport.2006.03.001

Burns, B. D. (2001). The hot hand in basketball: Fallacy or adaptive thinking? In J. D. Moore \& K. Stenning (Eds.), Proceedings of the Cognitive Science Society (pp. 152-157). Hillsdale, NJ: Erlbaum.

Burns, B. D. (2004). Heuristics as beliefs and as behaviors: The adaptiveness of the "hot hand." Cognitive Psychology, 48, 295-331. doi:10.1016/ j.cogpsych.2003.07.003

Burns, B. D., \& Corpus, B. (2004). Randomness and inductions from streaks: "Gambler's fallacy" versus "hot hand." Psychonomic Bulletin \& Review, 11, 179-184. doi:10.3758/BF03206480

Camerer, C. F. (1989). Does the basketball market believe in the 'hot hand? American Economic Review, 79, 1257-1261.

Carlson, K. A., \& Shu, S. B. (2007). The rule of three: How the third event signals the emergence of a streak. Organizational Behavior and Human Decision Processes, 104, 113-121. doi:10.1016/j.obhdp.2007.03.004

Caruso, E. M., \& Epley, N. (2004). Hot hands and cool machines: Perceived intentionality in the prediction of streaks. Paper presented at the 5th Annual Meeting of the Society for Personality and Social Psychology, Austin, TX.

Castaneda, J., \& Rodrigo, M. J. (1998). Developmental effects of the content of visually presented base rates. Current Psychology of Cognition, 3, 555-576.
Clark, R. D. (2003). An analysis of streaky performance on the LPGA tour Perceptual and Motor Skills, 97, 365-370.

Crothers, T. (1998). Texas tornadoes. Sports Illustrated, 88, 98-101.

Czienskowski, U. (2002). Hot-hand Program C++. Max Planck Institute for Human Development, Berlin, Germany.

Dawes, R. M. (1979). The robust beauty of improper linear models in decision making. American Psychologist, 34, 571-582. doi:10.1037/ 0003-066X.34.7.571

Dorsey-Palmateer, R., \& Smith, G. (2004). Bowlers' hot hands. The American Statistician, 58, 38-45. doi:10.1198/0003130042809

Gaissmaier, W., \& Schooler, L. J. (2008). The smart potential behind probability matching. Cognition, 109, 416-422. doi:10.1016/ j.cognition.2008.09.007

Gallistel, C. R. (1993). Organization of learning. Cambridge, MA: MIT Press.

Gigerenzer, G., Hell, W., \& Blank, H. (1988). Presentation and content: The use of base rates as a continuous variable. Journal of Experimental Psychology: Human Perception and Performance, 14, 513-525. doi: 10.1037/0096-1523.14.3.513

Gigerenzer, G., Todd, P. M., \& the ABC Research Group. (1999). Simple heuristics that make us smart. Oxford, England: Oxford University Press.

Gilden, D. L., \& Wilson, S. A. (1995a). On the nature of streaks in signal detection. Cognitive Psychology, 28, 17-64. doi:10.1006/ cogp.1995.1002

Gilden, D. L., \& Wilson, S. A. (1995b). Streaks in skilled performance. Psychonomic Bulletin \& Review, 2, 260-265. doi:10.3758/BF03210967

Gilovich, T. (1993). How we know what isn't so: The fallibility of human reason in everyday life. New York: The Free Press. ISBN 0-02911706-2.

Gilovich, T., Vallone, R., \& Tversky, A. (1985). The hot hand in basketball: On the misperception of random sequences. Cognitive Psychology, 17, 295-314. doi:10.1016/0010-0285(85)90010-6

Gould, D., Tammen, V., Murphy, S., \& May, J. (1991). An evaluation of U.S. Olympic sport psychology consultant effectiveness. The Sport Psychologist, 5, 111-127.

Gula, B., \& Köppen, J. (2009). Einfluss von Länge und Perfektion einer „Hot-Hand Sequenz auf Zuspielentscheidungen im Volleyball. [Influence of length and perfectionism of hot-hand sequences to playmakers allocations in volleyball]. Zeitschrift für Sportpsychologie, 16, 1-6. doi:10.1026/1612-5010.16.2.65

Gula, B., \& Raab, M. (2004). Hot hand belief and hot hand behavior: A comment on Koehler and Conley. Journal of Sport and Exercise Psychology, 26, 167-170.

Hahn, U., \& Warren, P. A. (2009). Perceptions of randomness. Why three heads are better than four. Psychological Review, 116, 454-461. doi: $10.1037 / \mathrm{a} 0015241$

Hales, S. (1999). An epistemologist looks at the hot hand in sports. Journal of the Philosophy of Sport, 25, 79-87.

Hertwig, R., \& Todd, P. M. (2003). More is not always better: The benefits of cognitive limits. In D. Hardman \& L. Macchi (Eds.), Thinking. Psychological perspectives on reasoning, judgment and decision making (pp. 213-231). Chichester, England: Wiley.

Kareev, Y. (2000). Seven (indeed, plus or minus two) and the detection of correlations. Psychological Review, 107, 397-402. doi:10.1037/0033295X.107.2.397

Klaasen, F. J. G. M., \& Magnus, J. R. (2001). Are points in tennis independent and identically distributed? Evidence from a dynamic binary panel data model. Journal of the American Statistical Association, 96, 500-509. doi:10.1198/016214501753168217

Koehler, J. J. (1996). The base rate fallacy reconsidered: Descriptive, normative, and methodological challenges. Behavioral and Brain Sciences, 19, 1-53. doi:10.1017/S0140525X00041157 
Koehler, J. J., \& Conley, C. A. (2003). The "hot hand" myth in professional basketball. Journal of Sport and Exercise Psychology, 25, 253-259.

Köppen, J., \& Raab, M. (in press). The hot and cold hand in volleyball: Individual expertise differences in a video-based playmaker decision test. The Sport Psychologist.

Laplace, P.-S. (1951). A philosophical essay on probabilities (F. W. Truscott \& F. L. Emery, Trans.). New York, NY: Dover. (Original work published 1814)

Larkey, P. D., Kadane, J. B., \& Smith, R. A. (2004). Elusive illusions: The endless search for hot hands, and streak shooters in basketball. Working paper.

Larkey, P. D., Smith, R. A., \& Kadane, J. B. (1989). It's okay to believe in the hot hand. Chance: New Directions for Statistics and Computing, $4,22-30$.

Lyons, K. (2003). Performance analysis for coaches: Game analysis. Sports Coach, 26, 30-31.

McCallum, J. (1993). Hot hand, hot head. Sports Illustrated, 78, 22-24.

Mueser, P. R., Cowan, N., \& Mueser, K. T. (1999). A generalized signal detection model to predict rational variation in base rate use. Cognition, 69, 267-312. doi:10.1016/S0010-0277(98)00072-9

Oskarsson, T., Van Boven, L., McClelland, G. H., \& Hastie, R. (2009). What's next? Judging sequences of binary events. Psychological Bulletin, 135, 262-285. doi:10.1037/a0014821
Paiement, M., Baudin, P., \& Boucher, J. (1993). Scouting and match preparation at the national and international level. International Volley Technical Bulletin, 2, 4-14.

Schooler, L., \& Hertwig, R. (2005). How forgetting aids heuristic inference. Psychological Review, 112, 610-628. doi:10.1037/0033-295X .112 .3 .610

Smith, G. (2003). Horse shoe pitchers' hot hands. Psychonomic Bulletin \& Review, 10, 753-758. doi:10.3758/BF03196542

Sundali, J., \& Croson, R. (2006). Biases in casino betting: The hot hand and the gambler's fallacy. Judgment and Decision Making, 1, 1-12.

Tsyska, T., Zielonka, P., Decey, R., \& Sawicki, P. (2007). Perception of randomness and predicting uncertain events. Thinking \& Reasoning, 14, 83-110. doi: $10.1080 / 13546780701677669$

Tversky, A., \& Gilovich, T. (1989). The cold facts about the "hot hand" in basketball. Chance, 2, 16-21.

Wilke, A., \& Barrett, H. C. (2009). The hot hand phenomenon as a cognitive adaption to clumped resources. Evolution and Human Behavior, 30, 161-169. doi:10.1016/j.evolhumbehav.2008.11.004

Wolff, A. (1998). No question. Sports Illustrated, 88, 70-72.

Received August 28,2009

Revision received July 7, 2011

Accepted August 23, 2011

\section{Members of Underrepresented Groups: Reviewers for Journal Manuscripts Wanted}

If you are interested in reviewing manuscripts for APA journals, the APA Publications and Communications Board would like to invite your participation. Manuscript reviewers are vital to the publications process. As a reviewer, you would gain valuable experience in publishing. The $\mathrm{P} \& \mathrm{C}$ Board is particularly interested in encouraging members of underrepresented groups to participate more in this process.

If you are interested in reviewing manuscripts, please write APA Journals at Reviewers@apa.org. Please note the following important points:

- To be selected as a reviewer, you must have published articles in peer-reviewed journals. The experience of publishing provides a reviewer with the basis for preparing a thorough, objective review.

- To be selected, it is critical to be a regular reader of the five to six empirical journals that are most central to the area or journal for which you would like to review. Current knowledge of recently published research provides a reviewer with the knowledge base to evaluate a new submission within the context of existing research.

- To select the appropriate reviewers for each manuscript, the editor needs detailed information. Please include with your letter your vita. In the letter, please identify which APA journal(s) you are interested in, and describe your area of expertise. Be as specific as possible. For example, "social psychology" is not sufficient_you would need to specify "social cognition" or "attitude change" as well.

- Reviewing a manuscript takes time (1-4 hours per manuscript reviewed). If you are selected to review a manuscript, be prepared to invest the necessary time to evaluate the manuscript thoroughly. 